\title{
The $\beta$-Adrenergic Receptors and the Control of Adipose Tissue Metabolism and Thermogenesis
}

\author{
Sheila Collins* ${ }^{\dagger}$ and Richard S. Surwit ${ }^{\dagger}$ \\ Departments of *Pharmacology and ${ }^{\dagger} p_{\text {sychiairy and Behavioral Sciences, }}$ \\ Duke University Medical Center, Durham, North Carolina 277J0
}

\begin{abstract}
The $\beta$-adrenergic receptors ( $\beta$ ARs) are members of the large family of $G$ protein-coupled receptors. There are three $\beta A R$ subtypes $\left(\beta_{1} A R, \beta_{2} A R \beta_{3} A R\right)$, each of which is coupled to $G \alpha$ and the stimulation of intracellular cAMP levels. While $\beta_{1} A R$ and $\beta_{2} A R$ are broadly expressed throughout tissues of the body, $\beta_{3} A R$ is found predominantly in adipocytes. Stimulation of the $\beta A R s$ leads to lipolysis in white adipocytes and nonshivering thermogenesis in brown fat. However, in essentially all animal models of obesity, the $\beta A R$ system is dysfunctional and the ability to stimulate lipolysis and thermogenesis is impaired. Nevertheless, we and others have shown that selective $\beta_{3} A R$ agonists are able to prevent or reverse obesity and the loss of $\beta A R$ expression and to stimulate thermogencsis. This chapter will review the current understanding of the role of the sympathetic nervous system and the adipocyte $\beta A R$ s in models of obesity; the physiologic impact of changes in $\beta A R$ expression on body composition and thermogenesis; and the regulation and unique properties of $\beta A R$ subtypes in brown and white adipocytes. The latter includes our recent discovery of novel signal transduction mechanisms utilized by $\beta_{3} A R$ to activate simultaneously the protein kinase $A$ and MAP kinase pathways. The impact of understanding these pathways and their potential role in modulating adaptive thermogenesis is discussed
\end{abstract}

\section{Thermogenesis: Control of Heat Generation and Body Composition}

\section{A. BROWN FAT THERMOGENESIS AND UNCOUPLING OF MITOCHONDRIAL RESPIRATION}

The rich and varied history of brown adipose tissue (BAT) as an anatomically discrete tissue type included speculations during the 17th century that it was part of the thymus and, a century later, that it was an endocrine organ involved in blood formation or a form of fat acting as a reservoir for certain nutrients (Lindberg, 1970). It wasn't until 1961 that BAT was proposed to be thermogenic (Ball and Jungas, 1961; Smith, 1961). Since that time, an immense body of work has shown that BAT is uniquely capable of responding to various environmental stimuli to generate heat from stored metabolic energy. In response to sympathetic 
nervous system (SNS) activation, noradrenergic stimulation of beta-adrenergic receptors ( $\beta A R s$ ) (with a lesser contribution from $\alpha 1 \mathrm{AR}$ ) triggers an orchestrated hyperplastic and hypertrophic expansion of BAT. There is also a dramatic increase in blood flow and recruitment of metabolic fuel (i.e., free fatty acids, glucose) to support a massive increase in oxidative metabolism (Bukowiecki et al., 1982; Géloën et al., 1992). A unique and critical element of this thermogenic machine was recognized to be the presence of a brown fat-specific mitochondrial uncoupling protein (UCP), originally called "thermogenin" (Cannon et al., 1982). This mitochondrial protein allows controlled proton "leakage" across the inner mitochondrial membrane, along its electrochemical potential gradient, for the purpose of heat generation at the expense of coupled adenosine triphosphate (ATP) production (Figure 1). Cloning of the gene for the brown fat UCP (now called UCP1) from rodents provided the opportunity to investigate the molecular mechanism of thermogenic uncoupling in mitochondria and regulation of the UCP gene and protein by hormonal and metabolic stimulation (Bouillaud et al., 1984; Kozak et al., 1994; Larose et al., 1996). Today, although there is still much debate concerning the specific mechanism by which UCP1 promotes proton movement (Garlid

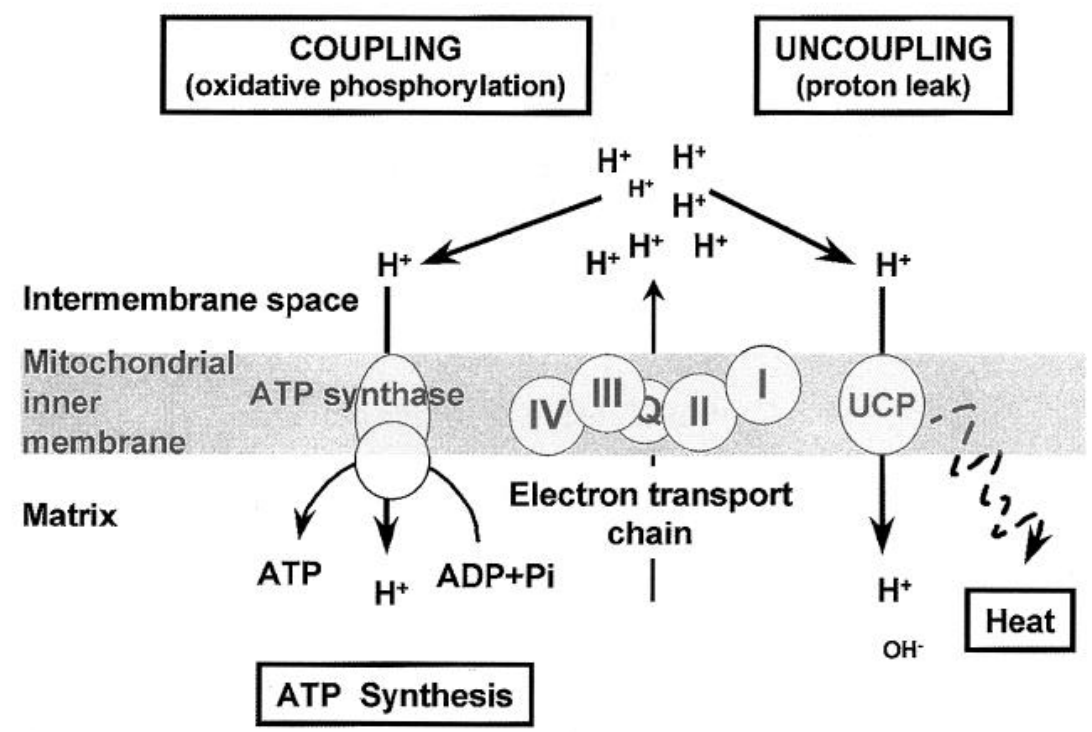

FIG. 1. Schematic model of mitochondrial "uncoupling." This simple cartoon compares the relationship between mitochondrial respiration that is "coupled" to the production of ATP via the ATP synthase, vs. the UCP-dependent transfer of protons across the inner membrane down the electrochemical gradient, whose action yields heat as a result of a need for increased fuel consumption to maintain ATP levels. 
et al., 1998; Bouillaud, 1999; Klingenberg et al., 1999), there is little dispute that this specialized member of the mitochondrial carrier family is responsible for the utilization of metabolic fuel to render mitochondrial metabolism inefficient, the byproduct of which is heat.

\section{B. THERMOGENIC DEFECTS IN OBESITY}

From the earliest studies of the ob/ob or "obese" mouse (now called leptindeficient $\mathrm{C} 57 \mathrm{BL} / 6 \mathrm{~J} L e p^{o b}$ ), there was evidence that these mice were not only obese, hyperglycemic, and hyperinsulinemic but that they also exhibited extreme sensitivity to the cold (reviewed in Bray and York, 1979). Histologically, BAT in these obese animals appears "inactive" in that it is infiltrated by white adipocytes and lacks the rich density of UCP1-containing mitochondria normally seen in lean animals (Cinti, 1999). The blunted capacity for adrenergic stimulation of lipolysis in adipose tissue of these animals (described in Section IIIA) probably also hinders the direct activation of UCP1 protein by free fatty acids. Other monogenic obesity models and hypothalamic lesioning studies in rodents all indicated a complex set of neural and endocrine abnormalitics, culminating in the loss of homeostatic mechanisms controlling both food intake and metabolic efficiency (reviewed in Bray et al., 1990).

The suggestive role for brown fat thermogenesis in body weight regulation was further strengthened by two sets of studies. First, mice lacking brown fat as a consequence of targeted expression of diptheria toxin in brown adipocytes (Lowell et al., 1993) became obese and somewhat hyperphagic. Since the adipocyte-derived hormone leptin (the product of the ob locus) (Zhang et al., 1994) regulates food intake, metabolic rate, and thermogenesis in brown fat (Campfield et al., 1995; Halaas et al., 1995; Pelleymounter et al., 1995; Collins et al., 1996), the obesity in brown fat-ablated mice may be a result of the inability of leptin to modulate brown fat thermogenesis. Second, Kopecky and Kozak showed that overexpression of UCPI in white adipose tissue (WAT) could ameliorate obesity syndromes resulting from either genetic or dietary manipulation (Kopecky et al., 1996a,1996b). In our studies, we have shown that high-fat feeding differentially increases plasma leptin, body temperature, and expression of UCP1 in BAT in the obesity-resistant A/J strain of mice (Surwit et al., 1997,1998). Since temperature is a surrogate measure for metabolic rate (Rising et al., 1992), this suggests that increased plasma leptin in response to high-fat feeding may serve to modulate the subsequent accumulation of body fat in rodents through increased metabolic activity in BAT. Recently, the notion that brown fat and UCP1 play a pivotal role in regulating body composition has been complicated by observations from specific targeted disruption of the UCP1 gene: these animals are completely intolerant of the cold, but are not obese (Enerback et al., 1997). Nevertheless, the inverse relationship between energy expenditure (particularly resting metabolic rate) and 
obesity has been observed repeatedly in laboratory animals and in humans (Ravussin et al., 1982; Landsberg and Young, 1984; Sims and Danforth, 1987; Spraul et al., 1993).

\section{UNUSUAL THERMOGENIC PROPERTIES OF ATYPICAL $\beta$ AR LIGANDS AND DISCOVERY OF THE $\beta_{3}$ AR}

\section{Studies in Laboratory Animal Models}

Study of lipolysis and thermogenesis in WAT and BAT by $\beta A R$ has witnessed episodic confusion and controversy, as the number of adrenergic receptor subtypes has grown by molecular cloning and new pharmacologic tools have become available. For many years, it was generally accepted that a single $\beta A R$ subtype controlled adipocyte metabolism (Fain and Garcia-Sainz, 1983; Bahouth and Malbon, 1988). This view began to erode as ever-more-selective sympathomimetic agents were developed that could discriminate between $\beta_{1} A R$ and $\beta_{2} A R$. Major evidence for a "new" $\beta A R$ subtype distinct from $\beta_{1}$ - or $\beta_{2} A R$ appeared when Arch and colleagues (1984) reported that a series of new $\beta$-adrenergic ligands that were clearly not "classical" $\beta_{1} A R$ or $\beta_{2}$ AR ligands had remarkable anti-obesity effects in the severely obese and diabetic C57BL/6J Lep ${ }^{o b}$ mouse. These novel compounds increased oxygen consumption, eliminated the classic cold intolerance of these animals, and, importantly, reduced their excess adipose tissue stores. In 1989 , the first report of a novel $\beta$ AR subtype, $\beta_{3} A R$, was published (Emorine et al., 1989), followed by reports of homologous genes in rodents (Granneman et al., 1991; Muzzin et al., 1991; Nahmias et al., 1991). It is now apparent that earlier results from $\beta A R$ radioligand-binding studies in adipose tissue were misleading, because the classical $\beta A R$ radioligands (e.g., cyanopindolol) exhibit a 20 - to 50 -fold weaker affinity for $\beta_{3} A R$ than for $\beta_{1} A R$ and $\beta_{2} A R$ (Tate et al., 1991). Therefore, $\beta_{3} A R$ levels were essentially undetected and any estimates made of $\beta_{1} A R$ and $\beta_{2} A R$ were distorted.

Many studies have documented the potent anti-obesity and antihyperglycemic properties of ever-more-selective $\beta_{3}$ AR agonists in a variety of animal models (Arch et al., 1984; Himms-Hagen et al., 1994; Largis et al., 1994; Collins and Surwit, 1996; Collins et al., 1997; Sasaki et al., 1998). In most species studied, including some studies in nonhuman primates, $\beta_{3}$ AR-agonist treatment is associated with increased density of brown adipocytes expressing UCP1 within typical WAT depots (Champigny et al., 1991; Himms-Hagen et al., 1994; Collins et al., 1997; Fisher et al., 1998; Sasaki et al., 1998). At least from our studies in various inbred strains of mice, the relative success of $\beta_{3}$ agonists as an anti-obesity therapy appears to depend upon genetic background and parallels the quantitative extent of this expansion of BAT (Collins et al., 1997). Others have reported similar 
effects of cold exposure as well as acute $\beta_{3}$-agonist stimulation in a series of recombinant inbred strains of mice (Guerra et al., 1998). Importantly, in our studies, we have observed, as shown in Figure 2A, that the beneficial effects of $\beta_{3} \mathrm{AR}$ agonists to decrease adipose tissue mass and improve glycemic control in mouse models of obesity and diabetes can persist, even after many weeks of chronic treatment (Collins et al., 1997). This apparent lack of functional desensitization is rather unusual, particularly since tachyphylaxis is a hallmark of most receptor systems. It is likely that $\beta_{3} A R$ activation and stimulation of downstream effectors can continue because $\beta_{3} A R$ is neither a target for phosphorylation (Liggett et al., 1993) nor does it bind $\beta$-arrestin (Cao et al., 2000; discussed in Section II), an accessory protein that is involved in desensitization of $\mathrm{G}$ protein-coupled receptors (Lefkowitz, 1998). In addition, as shown in Figure 2B, this decrease in body fat stores is not due to changes in food intake, dispelling the notion that these agents possess satiety properties.

\section{Controversy over the Existence of $\beta 3 A R$ in Humans}

The remarkable results from selective $\beta_{3} \mathrm{AR}$ agonist treatment in various laboratory animal models have fueled intense investigation of these drugs as potential obesity and/or diabetes therapies for humans. The presence of $\beta_{3} A R$ in adult human adipose tissue has been a subject of great debate. Part of the difficulty stems from the fact that $\beta_{3} A R$-selective agonists such as BRL37344 and CL316,243 initially were used to assess functional $\beta_{3} A R$ in fat samples from humans as well as nonhuman primates. The problem here is that these compounds are, at best, weak partial agonists at the primate homologues of $\beta_{3} \mathrm{AR}$ (Strosberg, 1997). Therefore, many studies with these compounds yielding negative results need to be carefully viewed in this light. There is now clear evidence of $\beta_{3} A R$ in human adipose tissue but the levels found in human fat cells are clearly much lower than those observed in rodents (Krief et al., 1993; Weyer et al., 1998) and are most evident in brown adipocytes found clustered amongst white fat cells, particularly within intra-abdominal depots. As a result of this visceral location, it has been difficult, to say the least, to quantify the relative abundance of these brown adipocytes among individuals and in response to drug treatments.

Humans are essentially like dogs and monkeys in that, as newborns, there are discrete depots of brown fat that "disappear" with growth into adulthood. However, upon treatment with selective $\beta_{3} A R$ agonists, dogs and rhesus monkeys have been found to exhibit increased metabolic rate, decreased fat mass, and the appearance of brown adipocytes scattered within the so-called typical "white" adipose depots, similar to what we and others have observed in mice and rats (Champigny et al., 1991; Fisher et al., 1998; Sasaki et al., 1998; Shih et al., 1999). These studies are promising in that they suggest that humans also may respond to 
$\mathbf{A}$

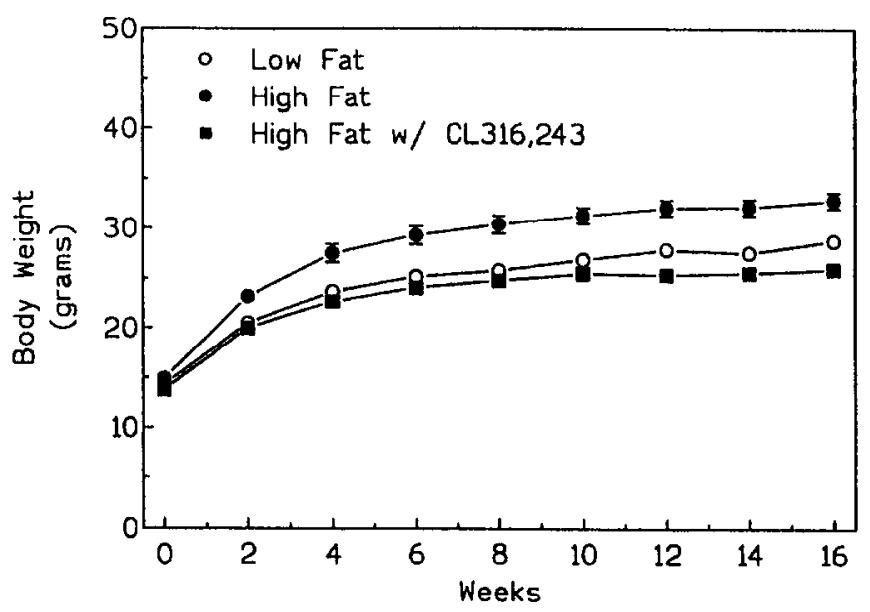

B

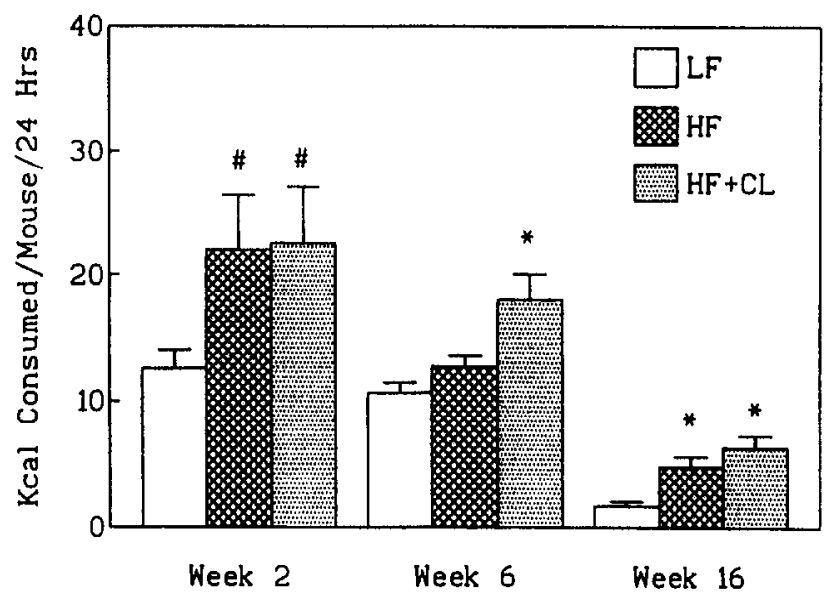

FIG. 2. Obesity induced by high-fat feeding and its prevention by a $\beta_{3} A R$ agonist. Thirty $A / J$ male mice were obtained from Jackson Laboratories (Bar Harbor, Maine) at 4 weeks of age. Groups of 10 mice were randomly assigned to a low-fat diet (open circles), high-fat diet (filled circles), or high-fat diet containing $0.001 \%$ CL316,243 (filled squares). (A) Animals were weighed at biweekly intervals throughout the 16 weeks on the diets. (B) Food intake measurements over a 24-hour period were made for individual animals at three different times during the study (after 2 weeks, 6 weeks, and 16 weeks on the diets). Each group of 10 animals was housed individually and grams of food consumed over 24 hours were measured. Caloric content of each diet was calculated based on 5.55 $\mathrm{kcal} / \mathrm{g}$ for the high-fat diets and $4.07 \mathrm{kcal} / \mathrm{g}$ for the low-fat diet. \#, significantly different from low-fat fed animals at $p<0.05 .^{*}$, significantly different from low-fat and high-fat animals at week 6 and from low-fat animals at week 16, p $<0.005$. [Adapted from Collins, S., and Surwit, R.S., J. Biol. Chem. 271, 9437-9440, 1996. Copyright 1996 The American Society for Biochemistry \& Molecular Biology.] 
these compounds. However, there is clearly a need for a more thorough, largescale analysis of intra-abdominal adipose tissue from humans, in order to obtain a more-accurate assessment of the extent to which brown adipocytes remain in adult humans. Likewise, the ability of newer compounds that are full agonists and antagonists for human $\beta_{3} \mathrm{AR}$ (Candelore et al., 1999) to directly modulate lipolysis in monkey or human fat cells should help to clarify these issues and to determine whether a similar recruitment of thermogenically active brown adipocytes will result from treatment of adult humans with $\beta_{3} \mathrm{AR}$ agonists.

\section{Why Three $\beta$ ARs in Adipocytes?}

The realization that adipocytes can express all three members of the $\beta A R$ family (van Liefde et al., 1992; Galitzky et al., 1993; Collins et al., 1994; Rohlfs et al., 1995), each coupled to the stimulation of adenylyl cyclase and the protein kinase A (PKA) pathway, raises the question as to whether these receptors are simply redundant or are responsible for regulating distinct cellular responses. Since the adipocyte-specific $\beta_{3}$ AR lacks sites for regulatory phosphorylation, a process that triggers rapid desensitization of most $G$ protein-coupled receptors, it was postulated that, during times of intense sympathetic stimulation, $\beta_{3} A R$ would remain functional while $\beta_{1} A R$ and $\beta_{2} A R$ became downregulated. However, even prior to discovery of $\beta_{3} A R$, studies in primary cultures of rodent brown adipocytes strongly suggested that $\beta A R$ subtypes may regulate discrete cellular functions in adipocytes. For example, $\beta_{1} \mathrm{AR}$ was shown to be linked to the proliferation of brown adipocytes in response to increased SNS stimulation (Bukowiecki et al., 1982; Géloën et al., 1992). Following the discovery of $\beta_{3} A R$ and the development of immortalized brown adipocyte cell systems (Kozak et al., 1992; Klaus et al., 1994), we examined the ability of individual $\beta A R$ subtypes to induce expression of UCP1 and to stimulate cell proliferation. We showed that induction of UCP1 transcripts could occur by any of the $\beta A R$ subtypes, although it appeared that the most-robust stimulation was achieved when the $\beta A R s$ were activated simultaneously (Rohlfs et al., 1995). By contrast, as shown in Figure 3, the norepinephrinestimulated proliferation and associated induction of c-fos and junB in B7 brown adipocytes were observed only in response to $\beta_{1} A R$ and, to a lesser extent, via $\beta_{2}$ AR but not via $\beta_{3}$ AR or $\alpha 1$ AR. As will be discussed in Section IIB, the ability of the $\beta A R s$ to couple to different signal transduction pathways, or to be physically localized in proximity to certain downstream targets, might contribute to nonoverlapping $\beta A R$ functions in the adipocyte. It should be noted that these immortalized brown adipocyte cell systems can provide certain significant advantages over primary cultures, particularly in terms of clonal purity and ease of growth. However, the maintenance of certain phenotypic characteristics - most notably, expression of UCP1 — can wane with passage (Irie et al., 1999). 


\section{Regulation and Signal Transduction Properties of the $\beta_{3} A R$}

\section{A. ADIPOCYTE-SPECIFIC EXPRESSION OF $\beta_{3}$ AR REQUIRES C/EBP $\alpha$}

Unlike $\beta_{1} A R$ and $\beta_{2} A R$, whose expression is quite ubiquitous, $\beta_{3} A R$ is confined to adipocytes. From studies in adipocyte cell lines - such as 3T3-F442A (Figure 4), 3T3-L1, and C3H10T1/2 - functional $\beta_{3} \mathrm{AR}$ appears relatively late during the process of adipogenesis (Feve et al., 1991); Dixon et al., in press). Two groups of transcription factors are responsible for initiating and maintaining adipocyte differentiation: CCAAT/enhancer binding protein (C/EBP) (Darlington et al., 1998) and peroxisome proliferator-activated receptor-gamma (PPAR $\gamma$ ) (Spiegelman, 1998). The C/EBPs are expressed in a cascade-like fashion during
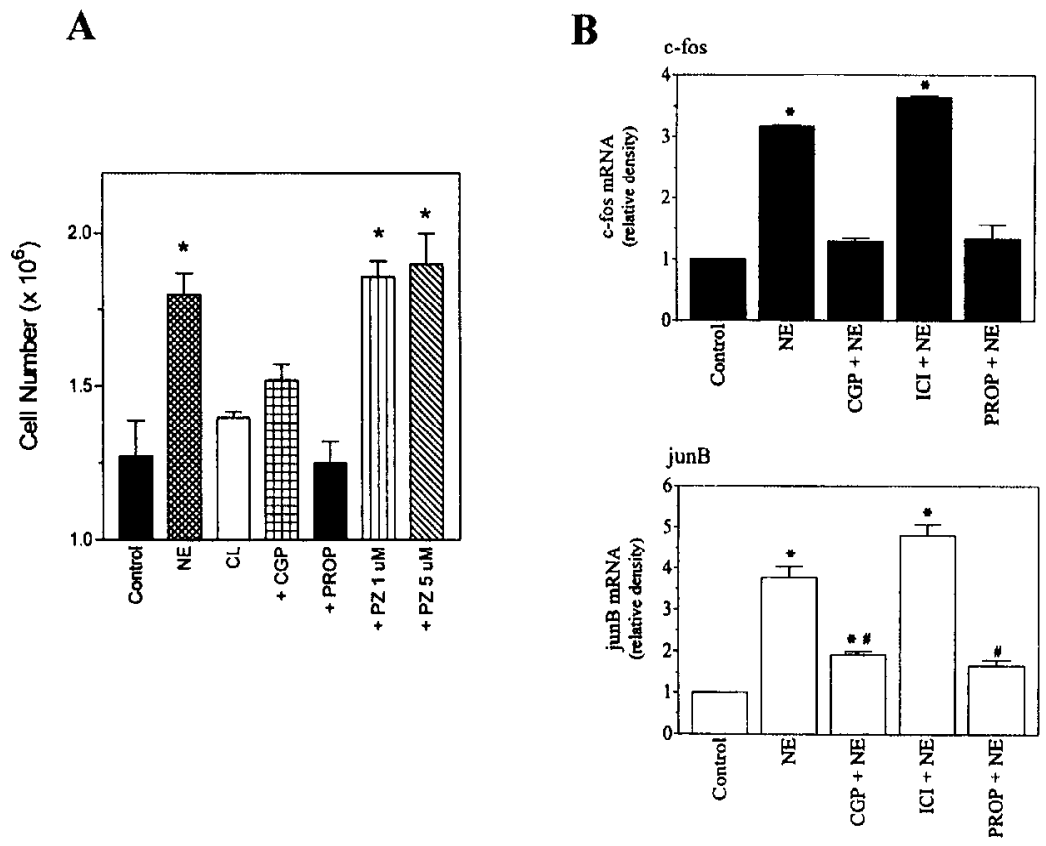

FIG. 3. Effect of selective activation of $\beta$-adrenergic receptor subtypes on proliferation and expression of fos/jun family members in $\mathrm{B} 7$ brown adipocytes. One day after seeding into six-well plates, the cells were treated with $1 \mu \mathrm{M} \mathrm{NE}$ or CL316,243 (CL) supplemented with or without $1 \mu \mathrm{M}$ of the following $\beta A R$ antagonists: CGP20712A (CGP) ICI118,551 (ICI) or propranolol (PROP), or the AR antagonist prazosin (PZ). Media with drugs were replaced every 2 days. (A) The data shown represent the cell number on day 6 (mean $\pm S . E . M . ; n=6$ ). ${ }^{*}$; values signiticantly different from untreated control $(p<0.001)$. All other groups were not different from the unstimulated control. (B) Cells were treated with NE $\pm \beta$ antagonists for 30 minutes. RNA was collected and analyzed by Northern blotting. RNA levels of cyclophilin served as a control for RNA/lane. *, $p<0.001$; \#, p < $0.05)$. 
the early stages of adipocyte differentiation, with C/EBP $\beta$ and C/EBP $\delta$ preceding the appearance of C/EBP $\alpha$ (Cao et al., 1991; Yeh et al., 1995). More recent studies indicate that the expression of PPAR $\gamma$ is partially under the control of the C/EBP family of transcription factors and vice versa (reviewed in Wu et al., 1999). By examining a series of adipogenic cell lines expressing various combinations of $\mathrm{PPAR} \gamma$ and $\mathrm{C} / \mathrm{EBPs}$, we find that $\mathrm{C} / \mathrm{EBP} \alpha$ is required for the adipocyte-dependent expression of the mouse $\beta_{3}$ AR gene (Figure 5). We have defined the C/EBP-binding site responsible for this regulation in the $\beta_{3} \mathrm{AR}$ promoter (Dixon et al., 2000).

\section{B. COUPLING OF $\beta_{3}$ AR IN ADIPOCYTES TO MULTIPLE SIGNALING PATHWAYS}

Long before the discovery of $\beta_{3} \mathrm{AR}$ and its recognition as a unique, adipocyte-specific receptor controlling lipolysis and thermogenesis, the Rodbell group made the observation that there was an unusual, biphasic stimulation of cyclic AMP (cAMP) production in adipocytes in response to the $\beta A R$ agonist isoproterenol (Cooper et al., 1979). Depending upon the concentration of guanosine triphosphate (GTP) in the assay, isoproterenol could either stimulate or inhibit adenylyl cyclase activity in adipocyte plasma membranes. Murayama and Ui (1983) showed that this inhibitory phase could be relieved by pretreating adipocytes with pertussis toxin (PTX). With the cloning of the $\beta_{3} \mathrm{AR}$ gene and the development of highly selective $\beta_{3} \mathrm{AR}$ agonists (Arch et al., 1984; Bloom et al.,

\section{Adenylyl Cyclase}

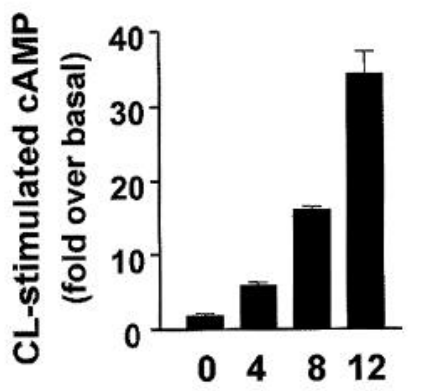

Days

\section{Northern Blot}

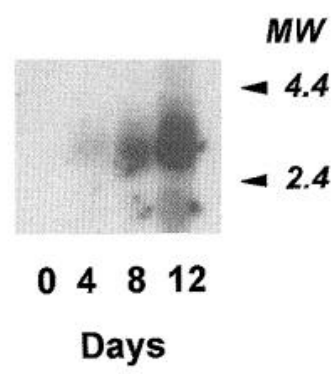

FIG. 4. Differentiation-dependent expression and activity of $\beta_{3} \mathrm{AR}$ in 3T3-F442A adipocytes. As adipogenic cell lines such as 3T3-F442A differentiate, the expression and functional activity of $\beta_{3} \mathrm{AR}$ appear. (Left panel) Cyclic AMP production in cells differentiated for the indicated periods. (Right panel) Northern blot analysis of $\beta_{3} \mathrm{AR}$ mRNA levels. 


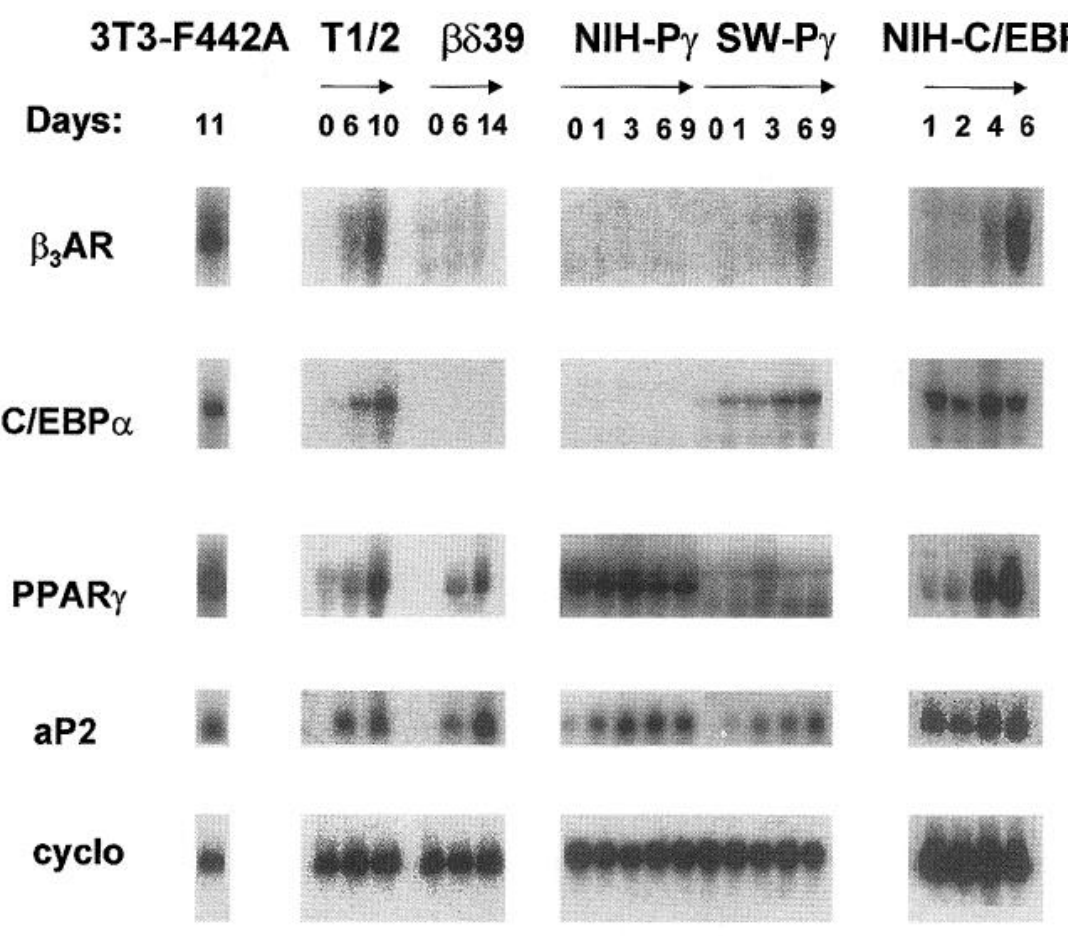

FIG. 5. Expression of $\beta_{3} A R$ during adipocyte differentiation is dependent on $C / E B P \alpha$. Cell lines indicated were differentiated and expression of $\operatorname{PPAR} \gamma, \mathrm{C} / \mathrm{EBP} \alpha$, the fatty acid binding protein aP2, and $\beta_{3}$ AR were examined by Northern blotting and adenylyl cyclase activity (not shown). Cyclophilin (cyclo) mRNA levels were monitored as a control for the amount of RNA applied to the gel.

1992), it was postulated that this novel, adipocyte-specific $\beta A R$ may be responsible for the biphasic adenylyl cyclase response in adipocytes (Begin-Heick, 1995). We previously noted that, despite the relatively high level of expression of $\beta_{3} \mathrm{AR}$ in adipocytes, the efficiency of coupling of $\beta_{3} \mathrm{AR}$ to stimulation of adenylyl cyclase is rather low (Collins et al., 1994). However, until recently, there had been no clear biochemical demonstration of physical coupling of $\beta_{3} \mathrm{AR}$ to $\mathrm{Gi}$, other than comparative functional experiments in the presence or absence of PTX. Nor had there been any indication of what additional second messenger pathway may be activated as a consequence of this putative coupling of $\beta_{3} \mathrm{AR}$ to Gi. We recently reported (Soeder et al., 1999) that $\beta_{3} A R$ is simultaneously coupled to $\mathrm{Gs}$ and $\mathrm{Gi}$, with the consequent activation of the PKA and mitogen-activated protein (MAP) kinase pathways, respectively (Figure 6). Physical coupling of $\beta_{3} \mathrm{AR}$ to $\mathrm{Gs}$ and $\mathrm{Gi}$ was demonstrated in cultured adipocytes using a photolabeling technique that relies upon the ability of agonist-activated receptor to trigger 
$\mathrm{G} \alpha$-subunit dissociation and binding of GTP. Thus, these data showed the restraining effect of Gi on cAMP production and the dependence of MAP kinase signaling on Gi activation by $\beta_{3} \mathrm{AR}$ (Soeder et al., 1999). More recent work in our laboratory shows that novel sequence elements within the $\beta_{3} \mathrm{AR}$ itself are responsible for the direct recruitment of $\mathrm{SH} 3$ domain-containing signaling molecules such as c-Src. This interaction is required to trigger the extracellular signalregulated kinase (ERK) cascade (Collins et al., 2000; Cao et al., 2000) (Figure 7). Further complicating this situation is the possibility that other members of the MAPK cascade may be involved.
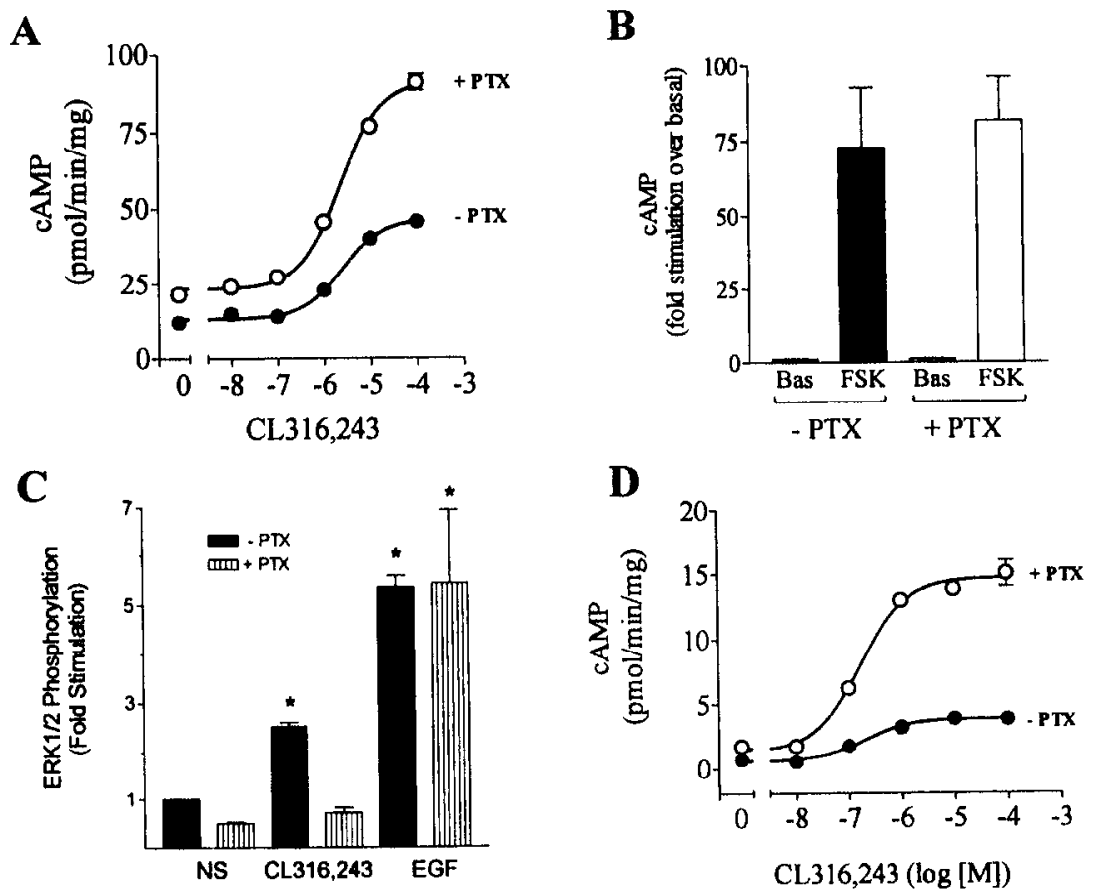

FIG. 6. $\beta_{3} \mathrm{AR}$ in adipocytes is coupled to $\mathrm{Gs}$ and $\mathrm{Gi}$, for stimulation of cAMP and MAP kinase. (A) Dose-response curve for agonist stimulation of cAMP production in differentiated 3T3-F442A adipocytes in response to CL316,243. Cells were pretreated or not with PTX, as indicated. (B) Basal and forskolin-stimulated cAMP production in membranes from untreated and PTX-pretreated cells. (C) Quantitation of ERK1/2 phosphorylation in response to CL316,243 $(5 \mu \mathrm{M})$ and EGF $(10 \mathrm{ng} / \mathrm{ml})$ after pretreatment with PTX, as indicated. *, one-way ANOVA, $p<0.001$. (D) Dose-response curve for agonist stimulation of cAMP production in CHO cells stably transfected with mouse $\beta_{3} \mathrm{AR}$ in response to CL316,243. Cells were pretreated or not with PTX, as indicated. [Adapted from Soeder, K.S., Snedden, K.S., et al., J. Biol. Chem. 274,12017-12022, 1999. Copyright 1999 The American Society for Biochemistry \& Molecular Biology.] 
This realization that $\beta_{3} \mathrm{AR}$ in adipocytes is coupled to multiple signaling pathways has potentially important implications for understanding the unique physiologic properties of selective agonists for this receptor. For example, the discoveries that hormone-sensitive lipase (HSL), a classic target of $\beta$ AR-mediated PKA activation, is phosphorylated by (an)other kinase (Anthonsen et al., 1998) and that HSL is probably not a single isozyme (Saltiel, 2000) require that we re-examine our thinking about how this regulation is achieved. In addition, the lipogenic transcription factor PPAR $\gamma$ can be phosphorylated by ERK1/2 on Ser 112. This modification serves to blunt its transcriptional activity (Hu et al., 1996; Adams et al., 1997). Alternatively, expression of the transcriptional coactivator PGC-1, which has been shown in model systems to stimulate mitochondriogenesis (Wu et al., 1999), is stimulated by cAMP (Puigserver et al., 1998). Thus, the possibility needs to be explored that the combined activation of the PKA and

A. Northern blot

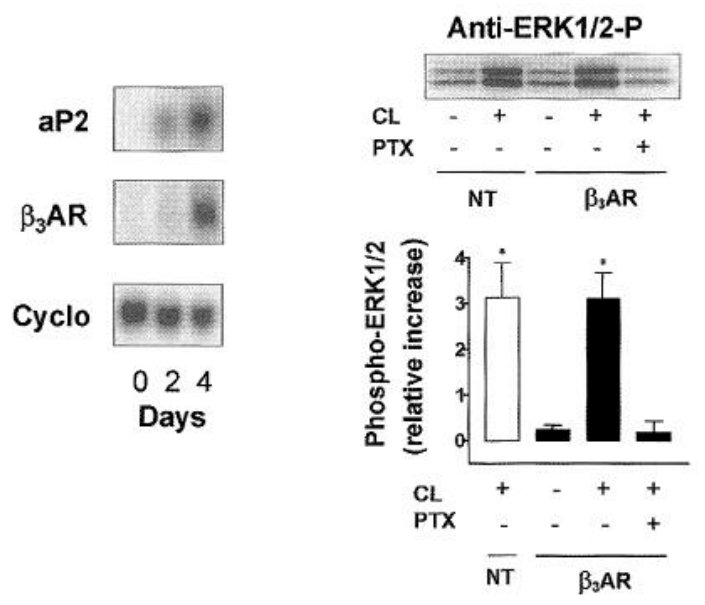

C. IP
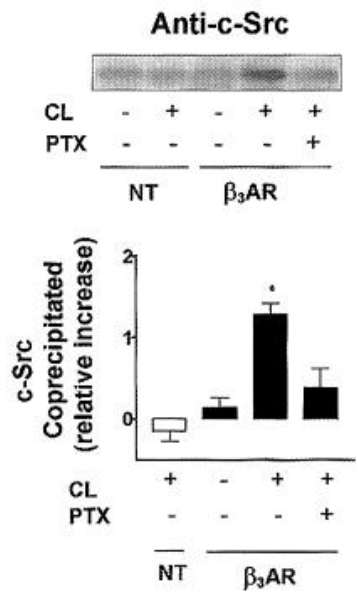

FIG. 7. $\beta_{3} \mathrm{AR}$ recruits c-Src in a Gi protein- and agonist-dependent manner in differentiated C3H10T1/2 adipocytes. C3H10T1/2 cells were transfected with HA- $\beta_{3} \mathrm{AR}$ and differentiated as described in "Experimental Procedures." Immunoprecipitation assays (IP) were performed with anti-HA antibody. (A) Expression of aP2 and $\beta_{3} \mathrm{AR}$ mRNA as a function of differentiation on the indicated days. Levels of aP2 are maximal by day 4. Cyclophilin RNA (cyclo) is a control in Northern blotting. (B) The levels of phosphorylated ERK1/2 (ERK1/2-P) in cell lysates and quantification of three independent experiments (mean $\pm S D$ ). (C) The level of c-Src co-precipitated with $\beta_{3} A R$ and quantification of three independent experiments (mean $\pm \mathrm{SD}$ ). [Reprinted with permission from Cao, W., Luttrell, L.M., Medvedev, A.V., Pierce, K.L., Daniel, K.W., Dixon, T.M., Lefkowitz, R.J., and Collins, S. J. Biol. Chem. 275, 38131-38134, 2000. Copyright 2000 The American Society for Biochemistry \& Molecular Biology.] 
MAPK pathways could underlie the $\beta_{3} A R$ agonist-dependent appearance of thermogenically active brown adipocytes and a change in energy balance away from lipid storage, although this is admittedly just speculation at this point.

\section{Regulation of Body Composition by Adipose Tissue $\beta$-Adrenergic Receptors}

\section{A. IMPAIRED $\triangle$ DIPOSE TISSUE $\triangle$ DRENERGIC SIGNALING IN OBESITY}

It was known for many years that obese C57BL/6J Lep $p^{o b}$ and C57KsJ Lep $R^{d b}$ mice exhibited a marked inability to effectively mobilize triglycerides from WAT and were unable to recruit BAT for thermogenesis in response to cold temperature. These deficiencies indicate that adrenergic mechanisms regulating metabolism in both white and brown fat are affected (discussed in Collins et al., 1994). While defects in SNS outflow have been shown to be associated with obesity in these models (Levin et al., 1983; Takeuchi et al., 1994), other experiments clearly indicated that there was impaired $\beta A R$ function at the level of the adipocyte itself, independent of the availability of catecholamines (Shepherd et al., 1977).

In the late 1970 s and early 1980 s, many investigators tried to determine the nature of the molecular defect in adipocytes from these obese animals. The components of the adrenergic signal transduction pathway (at least those which were known at the time; which did not include $\beta_{3} \mathrm{AR}$ ) were examined. Despite a severe blunting of $\beta$-adrenergic stimulation of cAMP production and lipolysis, the activity of adenylyl cyclase itself and other downstream effectors of the lipolytic process did not differ between lean and obese animals (Bégin-Heick and Heick, 1977; Shepherd et al., 1977). The discovery of $\beta_{3}$ AR as a adipocyte-specific $\beta$ AR subtype forced a re-evaluation of years of work on adipocyte adrenoceptors. Therefore, we examined the expression and functional activity of all three $\beta A R s$ in adipocytes from lean and genetically obese (C57BL/6J Lep $\left.{ }^{o b}\right)$ mice. Figure 8 shows the impaired ability of $\beta$ agonists to stimulate adenylyl cyclase activity in WAT from the $L e p^{o b}$ and the dramatic decrease in expression of $\beta_{3} A R$ as well as a 80 percent loss of $\beta_{1} A R$ in these obese mice, compared to lean littermates (Collins et al., 1994). In these studies, a series of detailed pharmacologic analyses allowed us determine the relative contributions of the three subtypes to the response and to conclude that these reductions in expression of specific $\beta A R$ subtypes are fully responsible for the defects in catecholamine-stimulated lipolysis and thermogenesis observed in the C57BL/6J Lep ${ }^{a b}$ mouse. Similar findings of depressed $\beta_{3}$ AR mRNA levels in the Zucker fatty (fa/fa) rat were reported by Muzzin et al. (1991) but their relationship to changes in the function of the receptor and tissue responses were not examined in that study. We extended these 

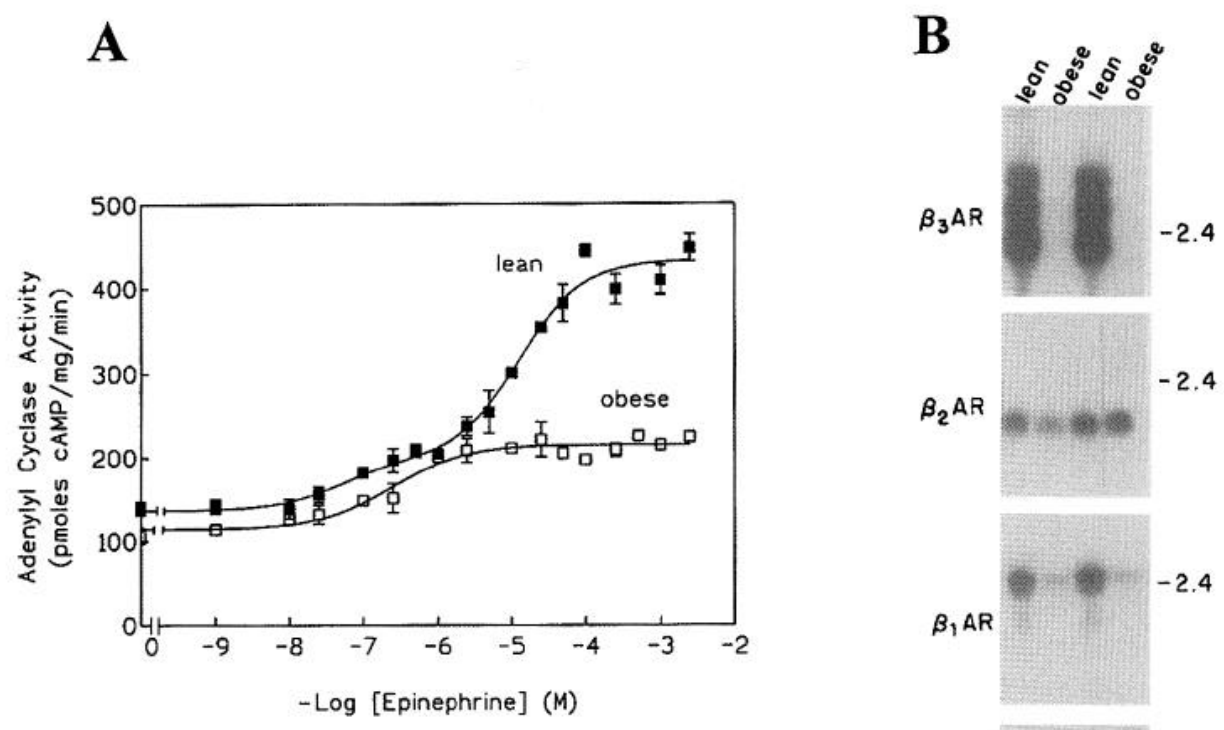

G3PDH $-1.4$

FIG. 8. $\beta$-agonist-stimulated adenylyl cyclase and expression of $\beta$ AR subtypes in WAT of C57BL/6J ("lean") and Lep ${ }^{o b}$ ("obese") mice. (Left panel) Dose-response curves of stimulation of cAMP production by epinephrine in WAT plasma membranes. (Right panel) Northern blot of $\beta_{1^{-}}, \beta_{2^{-}}$, and $\beta_{3} \mathrm{AR}$ mRNA levels in WAT. [Reprinted with permission from Collins, S., Daniel, K.W., et al., Mol. Endocrinol. 8, 518-527, 1994. Copyright The Endocrine Society.]

original findings in the $L e p^{o b}$ mouse to several other models of obesity. Significant deficits in the expression and function of adipocyte $\beta$ ARs were found in essentially every model of obesity that we have examined, including obesity induced by high-fat feeding in nonmutant mice (Collins et al., 1999) and in the slowly developed obesity associated with aging (Gettys et al., 1995).

The molecular basis for this dramatic impairment in $\beta A R$ expression and function may be related to the abnormal endocrine profiles observed in these models of obesity (reviewed in Bray et al., 1990). For example, in C57BL/6J $L e p^{o b}$ and C57BL/6J LepR $R^{d b}$ mice, hypercorticism is an important component in the development of obesity (Bray and York, 1979). However, manipulation of this system in the face of leptin deficiency is unable to fully reverse this phenotype. There is also significant adipose deposition in humans with Cushing's syndrome, particularly in intra-abdominal regions (Wilson et al., 1998). Despite this evidence for a role of the hypothalamic-pituitary-adrenal (HPA) axis in certain situations, most obese humans, as well as other mouse obesity mutants such as tubby and 
Cpe fat (Naggert et al., 1995; Noben-Trauth et al., 1996) and diet-induced obese mice (Surwit et al., 1988), have completely normal corticosteroid levels, yet clearly show reduced expression and function of adipocyte $\beta_{3}$ AR and $\beta_{1} A R$ (Collins et al., 1997,1999).

\section{B. OBESITY AND INSULIN RESISTANCE}

The single, most-common variable among all animal models of obesity, as well as a large percentage of obese humans, is the coexistence of hyperinsulinemia and insulin resistance. Due to this strong association between compromised glycemic control, obesity, and $\beta A R$ expression and function, we have proposed that this contributes, either directly or indirectly, to the inhibition of adipocyte $\beta \Lambda R$ expression and function (Collins et al., 1999). The result is impaired catecholamine-stimulated lipolysis, which exaggerates the excessive lipid storage in the adipocyte and consequent obesity. Evidence in support of this hypothesis includes the observation that $\beta_{3} A R$ expression rapidly declined in differentiated 3T3F442A mouse adipocytes treated with insulin (Fève et al., 1994). In addition, a role for insulin in affecting $\beta A R$ function in adipocytes is suggested by a series of studies showing that suppressing hyperinsulinemia with the $\mathrm{K}_{\text {ATP }}$ channel agonist, diazoxide, results in improved ability to stimulate lipolysis and a significant loss of adipose tissue mass (Alemzadeh et al., 1996,1998). The mechanism by which insulin may negatively impact $\beta A R$ function is not known but may be related to an insulin-dependent decrease in expression or function of $\mathrm{C} / \mathrm{EBP} \alpha$ (MacDougald et al., 1995), which we now know is critically required for proper expression of $\beta_{3} \mathrm{AR}$ in adipocytes (Dixon et al., 2000).

We provided additional support for the hypothesis that hyperinsulinemia directly affects adipocyte $\beta A R$ function in a recent study in which we used diazoxide to suppress diet-induced hyperinsulinemia in B6 mice. We observed a restoration of both $\beta_{3} \mathrm{AR}$ expression and function. Animals treated with diazoxide exhibit lower plasma insulin levels and increased cAMP production in adipocytes in response to a selective $\beta_{3} \mathrm{AR}$ agonist (Surwit et al., 2000). As a consequence of the restoration of $\beta_{3} A R$ expression by diazoxide, increased cAMP production was accompanied by significant loss of WAT mass. In combination with $\beta_{3} A R-$ agonist stimulation, there was a significant increase in UCP1 in brown fat. Because diazoxide could have some direct effects on $\mathrm{Ca}^{+2}$ influx through the adipocyte sulfonyl urea receptor 1 and $\mathrm{K}_{\mathrm{ATP}}$ to increase lipolysis (Shi et al., 1999), it is not clear to what extent the effects of diazoxide are due to decreased circulating insulin. In spite of this, the clinical efficacy is encouraging for future studies to explore the mechanisms responsible for these effects of diazoxide, with and without a selective $\beta_{3} A R$ agonist. 


\section{DISCOVERY OF UCP1 HOMOLOGS AND LINKAGE WITH METABOLIC FUEL HOMEOSTASIS}

Strong evidence of a proton "leak" in mitochondria from non-BAT tissues such as liver (Porter and Brand, 1993), coupled with sporadic observations that the brown fat UCP could be detected in other tissues such as muscle (Yoshida et al., 1994) (primarily when using antisera to measure protein levels), led Ricquier and colleagues to search for UCP homologues. In 1997, the isolation of UCP2, a novel gene with significant homology (59 percent) to the brown fat UCP (now named UCP1) was reported (Fleury et al., 1997). In addition to its ability to uncouple respiration as efficiently as UCP1 in model systems, UCP2 was broadly expressed in many tissues. These features of UCP2 led to the proposition that it was the long-sought explanation for the relative inefficiency of oxidative respiration seen in most cell types. In those initial studies, we noted that the UCP2 gene resides in a location on mouse chromosome 7 that is coincident with a quantitative trait locus (QTL) linked to hyperinsulinemia and high plasma leptin levels (reflective of body fat stores). In addition, we showed that consumption of a high-fat diet increased expression of UCP2 specifically in WAT in strains of mice that are relatively resistant to the development of diet-induced obesity and diabetes but not in obesity-prone strains of mice (Fleury et al., 1997; Surwit et al., 1998).This upregulation of UCP2 gene expression was postulated to be due to increased flux of fatty acids and a PPAR-dependent increase in UCP2 expression (Aubert et al., 1997; Camirand et al., 1998; Viguerie-Bascands et al., 1999). Subsequent discovcry of another UCP homologue, UCP3, which is expressed predominantly in skeletal muscle and brown fat, was reported by several groups (Boss et al., 1997; Vidal-Puig et al., 1997). The structural homology between these UCPs and basic features about their regulation and expression in various rodent models and human populations have been recently reviewed (Boss et al., 1998; Ricquier et al., 1999). Because the UCP3 gene is also located $8-10 \mathrm{~kb} \mathrm{5}$ to the UCP2 gene in both the mouse and human genomes (Solanes et al., 1997; Surwit et al., 1998), this close linkage relationship means that either or both (or neither) of these UCPs could be related to this QTL. However, we could find no evidence for changes, at least in expression of UCP3, in the mouse models that originally defined this QTL (Surwit et al., 1998). Confounding the concept that these novel UCPs may be involved in dissipation of excess caloric energy, it was reported that fasting led to increases, rather than decreases, in expression of UCP2 and UCP3. Dulloo and colleagues showed that blockade of the fasting-induced rise in free fatty acids completely prevented the increase in UCP2 and UCP3 mRNA (Samec et al., 1998,1999; Cadenas et al., 1999). Thus, in the aggregate, these novel UCPs would appear to be involved in the metabolic adaptations required during the fasted state, which requires a switch in fuel source from predominantly glucose to predominantly fatty acids. More importantly, since neither UCP2 nor UCP3 are expressed in 
hepatocytes under normal conditions, neither of these UCPs would appear to explain the well-documented proton leak in these cells (Porter and Brand, 1993).

Recently, several groups, including our own, have generated mice with targeted disruption of the UCP2 gene (Arsenijevic et al., 2000; Zhang et al., submitted) or the UCP3 gene (Gong et al., 2000; Vidal-Puig et al., 2000). At this point, there is no evidence of significant effects on either body temperature or body weight in either UCP2- or UCP3-deficient mice, even when challenged with a hyperlipidemic diet. However, since strain background can significantly affect the propensity to develop obesity and diabetes, the consequences of UCP2 or UCP3 deficiency may be revealed only under certain genetic or environmental situations. Nevertheless, novel phenotypes that are emerging in UCP2 -/- mice (Arsenijevic et al., 2000; unpublished observations) should help to shed light on the physiologic role of these mitochondrial proteins. Indeed, if we consider the fact that the mechanism of uncoupling by UCP1 is still debated many years after its discovery, perhaps we should not be surprised that efforts to determine the function of UCP2 and UCP3 at the physiologic and molecular level are unresolved.

\section{Summary}

During the past decade, the discovery of a host of new molecules involved in adipocyte metabolism (Friedman and Halaas, 1998), thermogenesis (Lowell and Spiegelman, 2000), and hypothalamic peptides and receptors (Schwartz et al., 2000 ) have led to new concepts and paradigms about the control of fuel intake and metabolism. While our understanding of the importance of catecholamines in controlling lipolysis and thermogenesis in the adipocyte is established, it is clear that we still have much work to do in order to understand the interplay between $\beta A R$ subtypes, their signaling pathways, and their regulation. Since pharmacologic treatments for obesity that focus on depressing food intake will likely never overcome the inevitable drop in energy expenditure that accompanies caloric restriction (Leibel et al., 1995), future discoveries in $\beta A R$ signaling mechanism and their targets in the adipocyte may provide not only insight into the "expenditure" side of the energy balance equation but also another therapeutic angle to exploit in the treatment of the growing obesity problem.

\section{ACKNOWLEDGMENTS}

This work was supported in part by National Institutes of Health grants R01DK53092 and R01DK54024 and an unrestricted gift from Novo Nordisk. We thank our collaborators and the members of our laboratories, which together have made this work possible. We also thank Claire Pecqueur for the original design of Figure 1. 


\section{REFERENCES}

Adams, M., Reginato, M., et al. (1997). J. Biol. Chem. 272(8), 5128-5132.

Alemzadeh, R., Jacobs, W., et al. (1996). Metabolism 45, 334-341.

Alemzadeh, R., Langley, G., et al. (1998). J. Clin. Endocrinol. Metab. 83, 1911-1915.

Anthonsen, M., Ronnstrand, L., et al. (1998). J. Biol. Chem. 273(1), 215-221.

Arch, J.R.S., Ainsworth, A.T., et al. (1984). Nature 309, 163-165.

Arsenijevic, D., Onuma, H., Pecqueur, C., Raimbault, S., Manning, B.S., Miroux, B., Couplan, E., Goubern, M., Alves-Guerra, M.-C., Surwit, R., Bouillard, F., Richard, D., Collins, S., and Ricquier, D. (2000). Nature Genet. 26, 435-439.

Aubert, J., Champigny, O., et al. (1997). Biochem. Biophys. Res. Comm. 238, 606-611.

Bahouth, S.W., and Malbon, C.C. (1988). Mol. Pharmacol. 34, 318-326.

Ball, E., and Jungas, R. (1961). Proc. Natl. Acad. Sci. U.S.A. 47, 932.

Begin-Heick, N. (1995). J. Cell. Biochem. 58, 464-473.

Bégin-Heick, N., and Heick, H.M.C. (1977). Can. J. Physiol. Pharmacol. 55, 1320-1329.

Bloom, J.D., Dutia, M.D., et al. (1992). J. Med. Chem. 35, 3081-3084.

Boss, O., Samec, S., et al. (1997). FEBS Lett. 408, 39-42.

Buss, O., Muzzin, P., et al. (1998). Eur. J. Endocrinol. 139, 1-9.

Bouillaud, F. (1999). Intl. J. Obes. 23(suppl. 6), S19-S23.

Bouillaud, F., Ricquier, D., et al. (1984). J. Biol. Chem. 259, 11583-11586.

Bray, G.A., and York, D.A. (1979). Physiol. Rev. 59, 719-809.

Bray, G.A., Fisler, J., et al. (1990). Front. Neuroendocrinol. 11, 128-181.

Bukowiecki, L., Collet, A., et al. (1982). Am. J. Physiol. 242, E353-E359.

Cadenas, S., Buckingham, J.A., et al. (1999). FEBS Lett. 462, 257-260.

Camirand, A., Marie, V., et al. (1998). Endocrinology 139, 428-431.

Campfield, L.A., Smith, F.J., et al. (1995). Science 269, 546-549.

Candelore, M.R., Deng, L., et al. (1999). J. Pharmacol. Exp. Ther. 290, 649-655.

Cannon, B., Hedin, A., et al. (1982). FEBS Lett. 150, 129-132.

Cao, Z., Umek, R.M., et al. (1991). Genes Devel. 5, 1538-1552.

Cao, W., Luttrell, L.M., Medvedev, A.V., Pierce, K.L., Daniel, K.W., Dixon, T.M., Lefkowitz, R.J., and Collins, S. (2000). J. Biol. Chem. 275, 38131-38134.

Champigny, O., Ricquier, D., et al. (1991). Proc. Natl. Acad. Sci. U.S.A. 88, 10774-10777.

Cinti, S. (1999). "The Adipose Organ." Editrice Kurtis, Milan, Italy.

Collins, S., and Surwit, R.S. (1996). J. Biol. Chem. 271, 9437-9440.

Collins, S., Daniel, K.W., et al. (1994). Mol. Endocrinol. 8, 518-527.

Collins, S., Kuhn, C.M., et al. (1996). Nature 380, 677.

Collins, S., Daniel, K.W., et al. (1997). Endocrinology 138, 405-413.

Collins, S., Daniel, K.W., et al. (1999). Intl. J. Obes. 23, 669-677.

Collins, S., Cao, W., et al. (2000). In "Adipocyte Biology and Hormone Signaling" (J.M. Ntambi, ed.), pp. 51-62. IOS Press, Washington, D.C.

Cooper, D., Schlegel, W., et al. (1979). J. Biol. Chem. 254, 8927-8931.

Darlington, G.J., Ross, S.E., et al. (1998). J. Biol. Chem. 273, 30057-30060.

Dixon, T.M., Daniel, K.W., Farmer, S.R., and Collins, S. (2000). J. Biol. Chem., in press.

Emorine, L.J., Marullo, S., et al. (1989). Science 245, 1118-1121.

Enerback, S., Jacobsson, A., et al. (1997). Nature 387, 90-94.

Fain, J., and Garcia-Sainz, J. (1983). J. Lipid Res. 24, 945-966.

Fève, B., Elhadri, K., et al. (1994). Proc. Nall. Acad. Sci. U.S.A. 91, 5677-5681.

Fisher, M.H., Amend, A.M., et al. (1998). J. Clin. Invest. 101, 2387-2393.

Fleury, C., Neverova, M., et al. (1997). Nature Genet. 15, 269-272.

Friedman, J.M., and Halaas, J.L. (1998). Nature 395, 763-770. 
Galitzky, J., Reverte, M., et al. (1993). Am. J. Physiol. 264, E403-E412.

Garlid, K.D., Jaburek, M., et al. (1998). FEBS Lett. 438, 10-14.

Géloèn, A., Collct, A.J., et al. (1992). Am. J. Physiol. 263, R1176-R1181.

Gettys, T.W., Rohlfs, E.M., et al. (1995). Endocrinology 136, 2022-2032.

Gong, D.W., Monemdjou, S., et al. (2000). J. Biol. Chem. 275, 16251-16257.

Granneman, J.G., Lahners, K.N., et al. (1991). Mol. Pharmacol. 40, 895-899.

Guerra, C., Koza, R.A., et al. (1998). J. Clin. Invest. 102, 412-420.

Halaas, J.L., Gajiwala, K.S., et al. (1995). Science 269, 543-546.

Himms-Hagen, J., Cui, J., et al. (1994). Am. J. Physiol. 266, R1371-R1382.

Hu, E., Kim, J.B., et al. (1996). Science 274, 2100-2103.

Irie, Y., Asano, A., et al. (1999). Biochem. Biophys. Res. Comm. 255, 221-225.

Klaus, S., Choy, L., et al. (1994). J. Cell Sci. 107, 313-319.

Klingenberg, M., Echtay, K.S., et al. (1999). Intl. J. Obes. 23(suppl. 6), S24-S29.

Kopecky, J., Hodny, Z., et al. (1996a). Am. J. Physiol. 270, E768-E775.

Kopecky, J., Rossmeisl, M., et al. (1996b). Am. J. Physiol. 270, E776-E786.

Kozak, U.C., Held, W., et al. (1992). Mol. Endocrinol. 6, 763-772.

Kozak, U.C., Kopecky, J., et al. (1994). Mol. Cell. Biol. 14, 59-67.

Krief, S., Lonnqvist, F., et al. (1993). J. Clin. Invest. 91, 344-349.

Landsberg, L., and Young, J.B. (1984). J. Clin. Endocrinol. Metab. 13, 475-499.

Largis, E.E., Burns, M.G., et al (1994). Drug Devel. Res. 32, 69-76.

Larose, M., Cassard-Doulcier, A.-M., et al. (1996). J. Biol. Chem. 271, 31533-31542.

Lefkowitz, R.J. (1998). J. Biol. Chem. 273, 18677-18680.

Leibel, R.L., Rosenbaum, M., et al. (1995). N. Engl. J. Med. 332, 621-628.

Levin, B.E., Triscari, J., et al. (1983). Am. J. Physiol. 244, R347-R371.

Liggett, S., Freedman, N.J., et al. (1993). Proc. Natl. Acad. Sci. U.S.A. 90, 3665-3669.

Lindberg, O. (1970). "Brown Adipose Tissue." American Elsevier Publishing Co., New York.

Lowell, B.B., and Spiegelman, B.M. (2000). Nature 404, 652-660.

Lowell, B.B., Susulic, V., et al. (1993). Nature 366, 740-742.

MacDougald, O.A., Cornelius, P., et al. (1995). J. Biol. Chem. 270, 647-654.

Murayama, T., and Ui, M. (1983). J. Biol. Chem. 258, 3319-3326.

Muzzin, P., Revelli, J.P., et al. (1991). J. Biol. Chem. 266, 24053-24058

Naggert, J.K., Fricker, L.D., et al. (1995). Nature Genet. 10, 135-142.

Nahmias, C., Blin, N, et al. (1991). EMBO J. 10,3721-3727.

Noben-Trauth, K., Naggert, J.K., et al. (1996). Nature 380, 534-538.

Pelleymounter, M.A., Cullen, M.J., et al. (1995). Science 269, 540-543.

Porter, R., and Brand, M. (1993). Nature 362, 628-630.

Puigserver, P., Wu, Z., et al. (1998). Cell 92, 829-839.

Ravussin, E., Burnand, B., et al. (1982). Am. J. Clin. Nutrit. 35, 566-573.

Ricquier, D., Fleury, C., et al. (1999). J. Intern. Med. 245, 637-642.

Rising, R., Keys, A., et al. (1992). Am. J. Physiol. 263, E730-E734.

Rohlfs, E.M., Daniel, K.W., et al. (1995). J. Biol. Chem. 270, 10723-10732.

Saltiel, A.R. (2000). Proc. Natl. Acad. Sci. U.S.A. 97, 535-537.

Samec, S., Seydoux, J., et al. (1998). FASEB J. 12, 715-724.

Samec, S., Seydoux, J., et al. (1999). Pfluger's Arch. 438, 452-457.

Sasaki, N., Uchida, E., et al. (1998). J. Vet. Med. Sci. 60, 465-469.

Schwartz, M., Woods, S., et al. (2000). Nature 404, 661-671.

Shepherd, R.E., Malbon, C.C., et al. (1977). J. Biol. Chem. 252, 7243-7248.

Shi, H., Moustaid-Moussa, N., et al. (1999). FASEB J. 13, 1833-1838.

Shih, T.L., Candelore, M.R., et al. (1999). Bioorg. Med. Chem. Lett. 9, 1251-1254.

Sims, E.A.H., and Danforth, E., Jr. (1987). J. Clin. Invest. 79, 1019-1025. 
Smith, R. (1961). Physiologist 4, 113.

Soeder, K.S., Snedden, S.K., et al. (1999). J. Biol. Chem. 274, 12017-12022.

Solanes, G., Vidal-Puig, A., et al (1997). J. Biol Chem. 272, 25433-25436.

Spiegelman, B. (1998). Diabetes 47, 507-514.

Spraul, M., Ravussin, E., et al. (1993). J. Clin. Invest. 92, 1730-1735.

Strosberg, A. (1997). Ann. Rev. Pharmacol. Toxicol. 37, 421-450.

Surwit, R.S., Kuhn, C.M., et al. (1988). Diabetes 37(9), 1163-1167.

Surwit, R., Petro, A., et al. (1997). Diabetes 46, 1516-1520.

Surwit, R.S., Wang, S., et al. (1998). Proc. Natl. Acad. Sci. U.S.A. 95, 4061-4065.

Surwit, R.S., Dixon, T.M., Petro, A., Daniel, K.W., and Collins, S. (2000). Endocrinology 141, 3630-3637.

Takeuchi, H., Matsuo, T., et al. (1994). J. Nutrit. 125, 920-925.

Tate, K.M., Briend-Sutren, M.-M., et al. (1991). Eur. J. Biochem. 196, 357-361.

van Liefde, I., van Witzenberg, A., et al. (1992). J. Pharmacol. Exp. Ther. 262, 552-558.

Vidal-Puig, A. (2000). Nature Genet. 26, 387-388.

Vidal-Puig, A., Solanes, G., et al. (1997). Biochem. Biophys. Res. Comm. 235, 79-82.

Vidal-Puig, A.J., Grujic, D., et al. (2000). J. Biol. Chem. 275, 16258-16266.

Viguerie-Bascands, N., Saulnier-Blache, J.S., et al. (1999). Biochem. Biophys. Res. Comm. 256, 138-141.

Weyer, C., Tataranni, P.A., et al. (1998). Diabetes 47, 1555-1561.

Wilson, J.D., Foster, D.W., et al. (eds.) (1998). "Williams Textbook of Endocrinology," edit 9. W.B. Saunders Company, Philadelphia.

Wu, Z., Puigserver, P., et al. (1999). Cell 98, 115-124.

Yeh, W.C., Cao, Z., et al. (1995). Genes Devel. 9, 168-181.

Yoshida, T., Sakane, N., et al. (1994). Life Sci. 54, 491-498.

Zhang, Y., Proenca, R., et al. (1994). Nature 372, 425-432. 